\title{
Diagnostic value of urinary exosomal miR-23b-3p, miR-30a-5p, and miR-151-3p in children with primary nephrotic syndrome
}

\author{
Dan Feng, Boying Wu, Yajiao Pang \\ Pediatric Department, Affiliated People's Hospital of Ningbo University, Ningbo, China \\ Contributions: (I) Conception and design: D Feng, B Wu; (II) Administrative support: Y Pang; (III) Provision of study materials or patients: D Feng, Y \\ Pang; (IV) Collection and assembly of data: All authors; (V) Data analysis and interpretation: D Feng, B Wu; (VI) Manuscript writing: All authors; (VII) \\ Final approval of manuscript: All authors. \\ Correspondence to: Dan Feng. Pediatric Department, Affiliated People's Hospital of Ningbo University; No. 251, Baizhang East Road, Yinzhou \\ District, Ningbo, China. Email: livia0123@163.com.
}

\begin{abstract}
Background: Primary nephrotic syndrome (NS) is a common disease of the urinary system with an unclear pathogenesis. We aimed to detect the levels of urinary exosomal miR-23b-3p, miR-30a-5p, and miR-151-3p in children with primary NS, and to explore their diagnostic value for NS.

Methods: A total of 115 patients with NS who were admitted to the hospital from June 2017 to June 2019 were selected as the observation group. According to the disease progression, they were divided into an active group (acute active phase, $\mathrm{n}=68$ ) and remission group (remission phase, $\mathrm{n}=47$ ). In all, 50 healthy children were selected as the control group. Levels of urinary exosomal miR-23b-3p, miR-30a-5p, and miR-151-3p of each group in different periods were detected.

Results: The 24-h urine protein, serum albumin (ALB), and serum total cholesterol (TC) levels were significantly higher in the observation group than in the control group $(\mathrm{P}<0.05)$, while those in the active group were significantly higher than those in the remission group $(\mathrm{P}<0.05)$. The levels of miR-23b-3p and miR-30a-5p were significantly higher in the observation group than in the control group, and significantly higher in the active group than in the remission group $(\mathrm{P}<0.05)$. No miR-151-3p was detected in the urinary exosomes of the two groups. After treatment, levels of exosomal miR-23b-3p and miR-30a-5p in the two groups both decreased significantly $(\mathrm{P}<0.05)$. Results of receiver operating curve $(\mathrm{ROC})$ curve analysis showed that urinary exosomal miR-23b-3p and miR-30a-5p can be used to identify children with NS and healthy children. The area under the ROC curve (AUC) was 0.711 for miR-23b-3p and 0.844 for miR-30a-5p.

Conclusions: The levels of miR-23b-3p and miR-30a-5p in urinary exosomes of children with NS were significantly higher than those in healthy children, and decreased significantly after treatment, indicating that miR-23b-3p and miR-30a-5p in urinary exosomes are potential indicators for diagnosing the progression of NS and monitoring the treatment effect.
\end{abstract}

Keywords: Urinary exosomes; miR-23b-3p; miR-30a-5p; miR-151-3p; primary nephrotic syndrome

Submitted Aug 26, 2020. Accepted for publication Sep 30, 2020.

doi: $10.21037 /$ tau-20-1260

View this article at: http://dx.doi.org/10.21037/tau-20-1260

\section{Introduction}

Primary nephrotic syndrome (NS) is a common urinary system disease of the pediatric population. In this condition, a variety of causes may lead to an increase in the permeability of the glomerular filtration membrane, resulting in the loss of a large amount of plasma protein and a series of symptoms, such as low proteinemia and hyperlipidemia (1). There are a variety of pathological types of NS, with micropathological type as a common type. Glucocorticoid therapy is the main means of clinical treatment of NS, but there are still some children who suffered from repeated attacks after treatment, or even 
progress to hormone-resistant NS, with poor therapeutic effect (2). Exosomes are membrane vesicles secreted by cells and widely exist in human body fluids. Exosomes contain specific proteins, lipids, nucleic acids and other substances, which are important carriers involved in cell communication and material transport (3). Some scholars believe that the overexpression of microRNA (miRNA) plays an important role in the progression of kidney disease, and the expression level of specific miRNA expression can reflect the severity of kidney disease (4). It has been found that miRNAs in humoral exosomes had high abundance and stability, while the expression of miRNAs in urine exosomes was closely related to the physiological function, pathological changes and treatment strategy selection of the kidney (5). At present, relevant studies have shown that certain miRNAs including miR-200a, miR-200b, and miR-29 play important roles in renal diseases like lupus nephritis and immunoglobulin A (IgA) nephropathy (6). However, few studies how examined the correlation between miR-23b-3p, miR-30a-5p, and miR-151-3p levels and the pathogenesis and progression of NS. Therefore, this study aimed to detect the levels of miR-23b-3p, miR-30a-5p, and miR-151-3p in the urine exosomes of children with NS, and to evaluate their application as auxiliary diagnostic indicators for NS. We present the following article in accordance with the STARD reporting checklist (available at http://dx.doi. org/10.21037/tau-20-1260).

\section{Methods}

\section{General information}

A total of 115 children with NS admitted to our hospital from June 2017 to June 2019 were selected and divided into an active group $(n=68)$ and a remission group $(n=47)$ according to the progress of the disease. The active group was the children with clinically confirmed NS, including 41 males and 27 females, ranging in age from 2 to 10 years old, with an average age of $5.87 \pm 3.42$ years old. As for disease type, there were 47 cases of the simple type and 21 cases of the nephritis type; for hormone reaction type, there were 48 cases of the sensitive type and 20 cases of the drug resistance type. The remission group consisted of children who improved and alleviated after comprehensive treatment with hormones, cytotoxic drugs and immunosuppressive agents, including 28 males and 19 females, ranging in age from 1 to 12 years old, with an average age of $6.15 \pm 3.18$ years old. As for disease type, there were 32 cases of the simple type and
15 cases of the nephritis type; for hormone reaction type, there were 35 cases of the sensitive type and 12 cases of the drug resistance type. All patients received hormone therapy and other adjuvant therapies including antihypertensive drugs, cyclophosphamide, tacrolimus etc. In addition, 50 healthy children who underwent physical examination during the same period were selected as the control group, including 22 males and 28 females, ranging in age from 2 to 10 years old, with an average age of $6.07 \pm 2.35$ years old. The study was approved by the hospital ethics committee, and differences in clinical baseline data between the groups were not statistically significant $(\mathrm{P}>0.05)$, indicating they were comparable.

The study was approved by the ethics committee of People's Hospital of Ningbo University (approval ID: 2020069). All the children's family members signed the informed consent. All procedures performed in this study involving human participants were in accordance with the Declaration of Helsinki (as revised in 2013).

\section{Inclusion and exclusion criteria}

The inclusion criteria for participants were the following: (I) clinical examination met the diagnostic criteria of NS (7), urine protein $>50 \mathrm{mg} / \mathrm{kg} / \mathrm{d}$; (II) aged $\leq 12$ years; (III) all family members signed the informed consent; (IV) no ingestion of drugs that could affect the test indicators. The exclusion criteria were the following: (I) patients diagnosed with secondary or congenital NS; (II) patients with severe urinary tract infection or other kidney diseases; or (III) patients with malignant tumors by examination.

\section{Extraction of total RNA from exosomes}

Morning urine $(8 \mathrm{~mL})$ was taken from children with NS and healthy children. After being centrifuged at room temperature to remove the cells, the supernatant was collected and stored in a refrigerator at $-80{ }^{\circ} \mathrm{C}$. The exosomes were extracted from the supernatant with an exosome extraction kit; and then the total RNA of the exosome was extracted using the Trizol method. After being dissolved by the addition of diethyl pyrocarbonate (DEPC), the RNA was stored in the refrigerator at $-80{ }^{\circ} \mathrm{C}$ until use.

\section{Real-time fluorescent quantitative polymerase chain reaction (RT-qPCR)}

TaqMan probe primers were used to detect the levels 
Table 1 Comparison of 24-h urinary protein, serum ALB, and TC levels in the three groups $\left(\bar{x}_{ \pm} \mathrm{s}\right)$

\begin{tabular}{|c|c|c|c|c|c|}
\hline Item & Active group & Remission group & Control group & $\mathrm{F}$ & $P$ \\
\hline 24-h urinary protein (g) & $6.23 \pm 1.23^{\star}$ & $5.46 \pm 1.58^{\star \#}$ & $0.84 \pm 0.27$ & 15.468 & $<0.001$ \\
\hline ALB (g/L) & $18.32 \pm 2.47^{\star}$ & $22.53 \pm 3.67^{\star^{\#}}$ & $32.75 \pm 3.81$ & 19.153 & $<0.001$ \\
\hline TC (mmol/L) & $12.78 \pm 3.06^{\star}$ & $10.46 \pm 3.41^{\star^{\#}}$ & $5.23 \pm 2.86$ & 12.476 & $<0.001$ \\
\hline
\end{tabular}

*, $\mathrm{P}<0.05$ compared with the control group; ${ }^{*}, \mathrm{P}<0.05$ compared with the remission group.

of miR-23b-3p, miR-30a-5p, and miR-151-3p in urine exosomes of children. The reverse transcription reaction system was $10 \mu \mathrm{L}$ of the total volume. After mixing, the mixture was put in the PCR machine to perform the reverse transcription reaction, with the reaction conditions of $16^{\circ} \mathrm{C}$ for $30 \mathrm{~min}, 42{ }^{\circ} \mathrm{C}$ for $30 \mathrm{~min}$, and $85^{\circ} \mathrm{C}$ for $5 \mathrm{~min}$; it was finally stored at $4{ }^{\circ} \mathrm{C}$. The reaction system of Real-time fluorescent quantitative PCR (RT-qPCR) was $20 \mu \mathrm{L}$ in a 96-well PCR plate. After mixing, the reaction conditions were performed at $95^{\circ} \mathrm{C}$ for 5 minutes, $95^{\circ} \mathrm{C}$ for 15 seconds, and $60^{\circ} \mathrm{C}$ for 1 minute, for a total of 40 cycles.

\section{Detection of 24 b-urine protein and serum indicators}

A dry chemical method was used to detect the urine protein level of children in the three groups. An automatic biochemical analyzer was used to detect the serum indicators, including albumin (ALB) and serum total cholesterol (TC), of children in the three groups.

\section{Statistical analysis}

Data were analyzed using SPSS 20.0 statistical software (IBM, Armonk, NY, USA). Measurement data are expressed as $(\bar{x} \pm s)$, and were analyzed using the single-factor analysis of variance among multiple groups. Least significant difference (LSD) $t$-test was used for multiple comparisons of the homogeneity of variance, and Tamhane's T2 test was used for the analysis of data with uneven variance. Measurement data of non-normal distribution are expressed as [M (P25-P75)], Mann-Whitney $\mathrm{U}$ test was used for the comparison between two groups, while the Kruskal-Wallis $\mathrm{H}$ test was used for comparison among multiple groups. Receiver operating curve (ROC) curve analysis was used to analyze the diagnostic value of urinary exosome indicators for NS. Results with a $\mathrm{P}$ value $<0.05$ were considered to be significantly different.

\section{Results}

Comparison of 2-b urine protein, serum ALB, and TC levels in the three groups

The levels of 24-h urine protein, serum ALB, and TC of NS children were significantly higher than those of the control group $(\mathrm{P}<0.05)$, while those of the active group were significantly higher than those in the remission group $(\mathrm{P}<0.05$, Table 1).

\section{Comparison of the exosomal levels of $m i R-23 b-3 p$, miR- 30a-5p, and miR-151-3p in the three groups}

miR-151-3p could not be detected in the urine exosomes of children with NS and healthy children. Interestingly, the levels of miR-23b-3p and miR-30a-5p in children with NS were significantly higher than those in the control group $(\mathrm{P}<0.05)$, and the levels of miR-23b-3p and miR-30a-5p in the active group were significantly higher than those in the remission group $(\mathrm{P}<0.05$, Table 2$)$.

\section{Comparison of exosomal miR-23b-3p and miR-30a-5p levels before and after treatment in children with NS}

Before treatment, there was no significant difference in the levels of exosomal miR-23b-3p and miR-30a-5p between the two groups of children $(\mathrm{P}>0.05)$. However, after treatment, the exosomal miR-23b-3p and miR-30a-5p of children in the two groups were significantly decreased $(\mathrm{P}<0.05$, Table 3).

\section{ROC curve analysis}

ROC curve analysis showed that detecting the levels of urine exosomal miR-23b-3p and miR-30a-5p can distinguish NS children from healthy children $(\mathrm{P}<0.05)$. The area under the curve (AUC) of miR-23b-3p was 0.711 
Table 2 Comparison of exosomal miR-23b-3p, miR-30a-5p, and miR-151-3p levels in the three groups [M ( $\left.\left.\mathrm{P}_{25}-\mathrm{P}_{75}\right), \mathrm{fmol} / \mathrm{L}\right]$

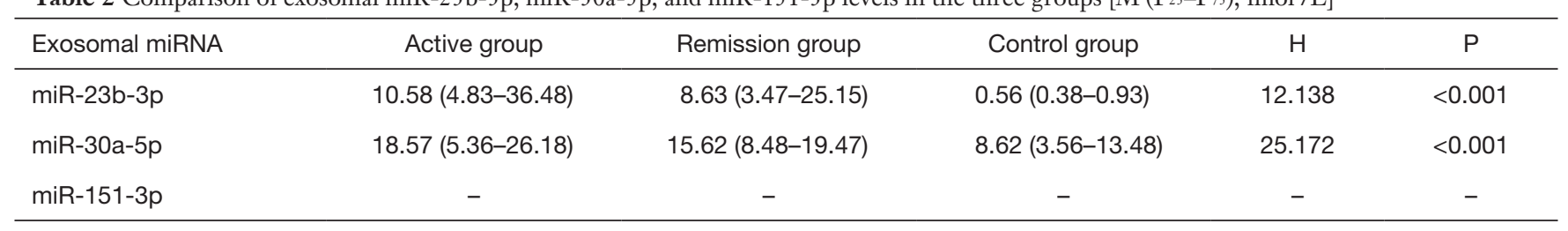

"_" indicates undetected level.

Table 3 Comparison of exosomal miR-23b-3p and miR-30a-5p levels before and after treatment in NS children $\left[\mathrm{M}\left(\mathrm{P}_{25}-\mathrm{P}_{75}\right)\right.$, fmol/L]

\begin{tabular}{lccr}
\hline miRNA level & Active group & Remission group & H \\
\hline miR-23b-3p & & & \\
Before treatment & $10.58(4.83-36.48)$ & $8.63(3.47-25.15)$ & 0.876 \\
After treatment & $2.68(1.52-5.03)^{\star}$ & $2.43(1.27-4.82)^{\star}$ & 4.582 \\
miR-30a-5p & & & $<0.001$ \\
Before treatment & $18.57(5.36 \sim 26.18)$ & $15.62(8.48 \sim 19.47)$ & 0.421 \\
After treatment & $10.48(6.68-15.57)^{\star}$ & $9.63(5.54-13.42)^{\star}$ & 3.517 \\
\hline
\end{tabular}

${ }^{*}, \mathrm{P}<0.05$ compared with the same group before treatment.

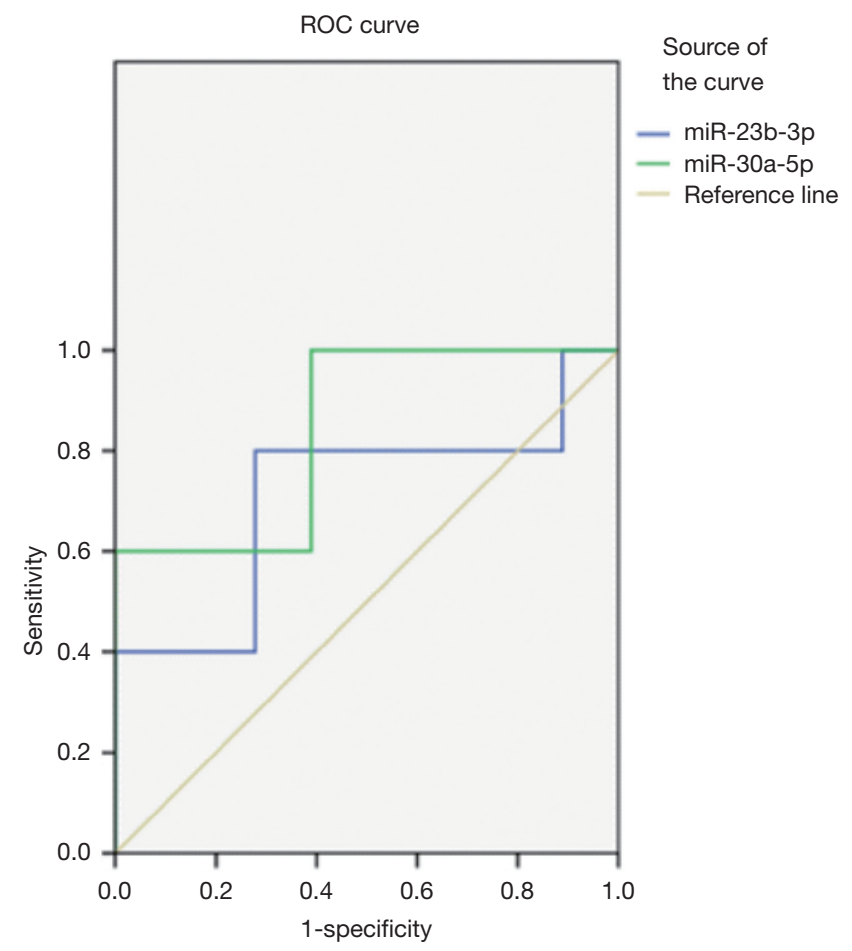

Figure 1 Receiver Operating Characteristic Curve (ROC) curve analysis of urine exosomal miR-23b-3p and miR-30a-5p in NS children in the active group. ROC, receiver operating curve; NS, nephrotic syndrome. and that of miR-30a-5p was 0.844 (Figure 1, Table 4).

\section{Discussion}

At present, the clinical diagnosis and monitoring of NS is mainly depended on renal biopsy. However, renal biopsy is a kind of traumatic examination, and children, due to their young age, can be fearful of the examination process, which in turn may affect the detection results (8). Therefore, it is of great significance for the diagnosis and treatment of NS to select non-invasive examination means to reflect the pathophysiological status of the kidney (9). Exosomes are a type of biologically active microvesicle secreted from cells, which can mediate the exchange of substances inside and outside of the cells (10). The exosomes in urine come from kidney tissue, the miRNAs in which are quite stable. The detection of miRNA in the exosomes can effectively reflect the kidney status of patients and evaluate the therapeutic effect. A variety of studies have shown that the expression level of miRNA is related to the pathological process of kidney disease, which may be a marker for the diagnosis of kidney disease. Lorenzen et al. (11) reported that the levels of miR-16 and miR-320 were downregulated in patients with acute kidney injury, while the level of miR-210 was significantly increased, and interpreted these as noninvasive 
Table 4 Comparison of the diagnostic efficacy of urine exosomes miR-23b-3p and miR-30a-5p for NS

\begin{tabular}{lcccccc}
\hline Indicator & AUC & $P$ & $95 \% \mathrm{Cl}$ & Younden's index & Sensitivity (\%) & Specificity (\%) \\
\hline miR-23b-3p & 0.786 & $<0.001$ & $0.666-0.878$ & 0.591 & 92.73 & 59.09 \\
miR-30a-5p & 0.780 & $<0.001$ & $0.660-0.873$ & 0.446 & 85.71 & 63.64 \\
\hline
\end{tabular}

molecular markers for monitoring the progression of kidney disease. Huang et al. (12) have confirmed that the expression level of urinary exosomal miRNA-193a is significantly increased in focal segmental glomerulosclerosis (SGS), which can assist in the diagnosis of the progression of SGS as a potential diagnostic marker for kidney disease.

The results in this study showed that the 24-h urine protein, serum ALB, and TC levels of NS children were significantly higher than those of the control group, and the 24-h urine protein, serum ALB, and TC levels of the active group were significantly higher than those of the remission group. This indicates that the levels of 24-h urine protein and serum indicators of NS children are significantly higher than those of healthy children, and the more severe the disease progression, the higher the 24-h urine protein and serum indicator levels are. Due to the disorder of the immune regulation mechanism and the increase of glomerular filtration rate, the glomerular filtration rate in children with NS is increased, resulting in the significant increase of ALB content in the urine. At the same time, the disorder of lipid metabolism in NS children also causes the abnormal increase of TC level. This can explain why the levels of 24-h urine protein, serum ALB, and TC of NS children in our study were significantly higher than those of healthy children (13). The comparison of urine exosomal miR-23b-3p and miR-30a-5p levels of children in the three groups revealed that the levels of miR-23b-3p and miR-30a-5p of NS children in the observation group were significantly higher than those in the control group; After treatment, the levels of exosomal miR-23b-3p and miR-30a-5p in NS children were both significantly decreased compared to those before treatment, suggesting that the levels of miR-23b-3p and miR-30a-5p change with the development of the disease. Therefore, the levels of exosomal miR-23b-3p and miR-30a-5p can effectively reflect the progress of the disease and monitor the treatment effect.

However, the molecular mechanism underlying miRNA expression in kidney disease is still unclear. Some researchers have proposed that the pathogenesis of NS is related to immune disorders and changes of glomerular permeability, as well as the abnormal expression of miRNA, which can also damage kidney function. Ho et al. (14) have found that miR-23b-3p is expressed in podocytes, and the lack of miR-23b-3p can affect the integrity of the glomerular basement membrane and cause damage to the renal tubules. In addition, the signaling pathway mediated by miR-23b-3p can down-regulate the expression of target gene Hesl, thereby inhibiting the Notch signaling pathway in monocyte formation and participating in the process of glomerulopathy $(15,16)$. In this study, the level of exosomal miR-23b-3p in children with NS was significantly higher than that in healthy children, which might because that the injury of glomerular podocytes in children with NS results in the upregulation of miR-23b-3p in urine exosomes. miR-30a-5p is also reported to be one of the miRNAs involved in the functional regulation of podocytes, which can affect the structure and function of podocytes by regulating the transcription factor (Xliml/Lhxl). After the lost of miR-30a-5p, injury of podocytes is aggravated and renal function is affected (17), which may be the reason for the changes in the level of urine exosome miR-30a-5p in children with NS in this study In this study, miR-151-3p could not be detected that is different to previous research results (18), which may be related to its lower expression level in the urine exosomes of children and the difference of detection methods. ROC curve analysis showed that urine exosomal miR-23b-3p and miR-30a-5p levels can distinguish NS children from healthy children. The AUC in both groups were over 0.5 , indicating that miR-23b-3p and miR-30a -5 p can be used as a potential molecular marker for NS diagnosis, which combined with conventional clinical indicators may improve the diagnostic efficiency of flat NS.

In summary, the levels of miR-23b-3p and miR-30a-5p in the urine exosomes of children with NS were significantly higher than those of healthy children, and their levels decreased significantly after treatment, indicating that miR-23b-3p and miR-30a-5p in urine exosomes are potential indicators for diagnosing the progress of NS and monitoring the effect of treatment. Due to the limitation of conditions, there are also some limitations that the sample 
size is small, and there is no further analysis of the influence of different urine exosome miRNA expression levels on NS treatment selection, which needs to be further studied and verified by further expanding the sample size.

\section{Acknowledgments}

Funding: None.

\section{Footnote}

Reporting Checklist: The authors have completed the STARD reporting checklist. Available at http://dx.doi.org/10.21037/ tau-20-1260

Data Sharing Statement: Available at http://dx.doi. org/10.21037/tau-20-1260

Conflicts of Interest: All authors have completed the ICMJE uniform disclosure form (available at http://dx.doi. org/10.21037/tau-20-1260). The authors have no conflicts of interest to declare.

Ethical Statement: The authors are accountable for all aspects of the work in ensuring that questions related to the accuracy or integrity of any part of the work are appropriately investigated and resolved. The study was approved by the ethics committee of People's Hospital of Ningbo University (approval ID: 2020-069). All the children's family members signed the informed consent. All procedures performed in this study involving human participants were in accordance with the Declaration of Helsinki (as revised in 2013).

Open Access Statement: This is an Open Access article distributed in accordance with the Creative Commons Attribution-NonCommercial-NoDerivs 4.0 International License (CC BY-NC-ND 4.0), which permits the noncommercial replication and distribution of the article with the strict proviso that no changes or edits are made and the original work is properly cited (including links to both the formal publication through the relevant DOI and the license). See: https://creativecommons.org/licenses/by-nc-nd/4.0/.

\section{References}

1. Wang CS, Greenbaum LA. Nephrotic Syndrome. Pediatr Clin North Am 2019;66:73-85.
2. Kodner C. Diagnosis and Management of Nephrotic Syndrome in Adults. Am Fam Physician 2016;93:479-85.

3. Huang Z, Zhang Y, Zhou J, et al. Urinary Exosomal miR193a Can Be a Potential Biomarker for the Diagnosis of Primary Focal Segmental Glomerulosclerosis in Children. Biomed Res Int 2017;2017:7298160.

4. Liu Y, Liu B, Liu Y, et al. MicroRNA expression profile by next-generation sequencing in a novel rat model of contrast-induced acute kidney injury. Ann Transl Med 2019;7:178.

5. Kapogiannis D. Exosome Biomarkers Revolutionize Preclinical Diagnosis of Neurodegenerative Diseases and Assessment of Treatment Responses in Clinical Trials. Adv Exp Med Biol 2020;1195:149.

6. Xia X, Wang Y, Huang Y, et al. Exosomal miRNAs in central nervous system diseases: biomarkers, pathological mediators, protective factors and therapeutic agents. Prog Neurobiol 2019;183:101694.

7. McCloskey O, Maxwell AP. Diagnosis and management of nephrotic syndrome. Practitioner 2017;261:11-5.

8. Souilmi FZ, Houssaini TS, Alaoui H, et al. Indications and results of renal biopsy in children: A single-center experience from Morocco. Saudi J Kidney Dis Transpl 2015;26:810-5.

9. Najafian B, Mauer M, Hopkin RJ, et al. Renal complications of Fabry disease in children. Pediatr Nephrol 2013;28:679-87.

10. Zhang J, Li S, Li L, et al. Exosome and exosomal microRNA: trafficking, sorting, and function. Genomics Proteomics Bioinformatics 2015;13:17-24.

11. Lorenzen JM, Kielstein JT, Hafer C, et al. Circulating miR-210 predicts survival in critically ill patients with acute kidney injury. Clin J Am Soc Nephrol 2011;6:1540-6.

12. Huang $Z$, Zhang $Y$, Zhou J, et al. Urinary Exosomal miR193a Can Be a Potential Biomarker for the Diagnosis of Primary Focal Segmental Glomerulosclerosis in Children. Biomed Res Int 2017;2017:7298160.

13. Barkai L, Soós A, Vámosi I. The effect of captopril therapy on the degree of microalbuminuria in diabetic children and adolescents with incipient nephropathy. Orv Hetil 1996;137:2565-8.

14. Ho J, Ng KH, Rosen S, et al. Podocyte-specific loss of functional microRNAs leads to rapid glomerular and tubular injury. J Am Soc Nephrol 2008;19:2069-75.

15. Tang Y, Yan W, Sun L, et al. Prediction of intravenous immunoglobulin resistance in Kawasaki disease in an East China population. Clin Rheumatol 2016;35:2771-6. 
16. Kimura H, Kawasaki H, Taira K. Mouse microRNA-23b regulates expression of Hes1 gene in P19 cells. Nucleic Acids Symp Ser (Oxf) 2004;(48):213-4.

17. Agrawal R, Tran U, Wessely O. The miR-30 miRNA family regulates Xenopus pronephros development and targets the transcription factor Xlim1/Lhx1. Development
2009;136:3927-36.

18. Luo Y, Wang C, Chen X, et al. Increased serum and urinary microRNAs in children with idiopathic nephrotic syndrome. Clin Chem 2013;59:658-66.

(English Language Editor: J. Gray)

Cite this article as: Feng $\mathrm{D}, \mathrm{Wu} \mathrm{B}$, Pang Y. Diagnostic value of urinary exosomal miR-23b-3p, miR-30a-5p, and miR-151$3 \mathrm{p}$ in children with primary nephrotic syndrome. Transl Androl Urol 2020;9(5):2235-2241. doi:10.21037/tau-20-1260 